

\title{
Aproximación normativa de protección de datos y los derechos de los menores de edad en Colombia
}

\section{Resumen}

El presente artículo tiene como objetivo problematizar algunos retos que tiene la sociedad contemporánea, en especial la dispuesta en Colombia, en torno a la preservación de los derechos que tienen los menores de edad ante el acceso y uso de los diferentes dispositivos y recursos tecnológicos que hacen parte el mundo digital actual. Para tal fin, este artículo toma como base algunos referentes expuestos por autores que han venido abordando el tema aquí propuesto, además de algunos ejemplos del marco normativo vigente en Colombia, como recursos que permitirán, no solo, sentar las bases argumentativas de este ensayo, sino también el horizonte propositivo explícito en él, a saber: la necesidad de avanzar en el desarrollo de un marco normativo más articulado y orientado a la prevención de la vulneración de los derechos que, por desconocimiento u otros motivos, se vulneran en los menores de edad en la actualidad en Colombia, así como de la necesidad de una mayor integración y promoción de un escenario de coresponsabilidad de todos los agentes sociales vinculados al proceso de formación de los menores de edad, tanto fuera como dentro del contexto educativo formal, en favor de garantizar un marco social que acompañe y vele eficientemente por los derechos de la población tomada como centro de nuestro debate.

Palabras clave: Protección de datos; Redes sociales; Cyberbulling; Menores de edad; Colombia.

\author{
Elias Said-Hung \\ Professor na Universidade do \\ Norte - Barranquilla - Colômbia. \\ Doutor em Ciências da \\ Informação pela Universidade \\ Complutense de Madrid - \\ Espanha \\ saide@uninorte.edu.co
}

Carlos Montoya Lemus

Graduado pela Universidade do Norte - Barranquilla - Colômbia. Cientista Político com ênfase em Governo e Políticas Públicas. Especialista em Projetos de

Desenvolvimento lemusc@uninorte.edu.co

\section{Francisco Durán Ruiz}

Professor na Universidade de Granada - Espanha. Doutor em Direito pela Universidade de Bologna - Itália fduranr@ugr.es

\section{Para citar este artigo:}

SAID-HUNG, Elias; LEMUS, Carlos Montoya; RUIZ, Francisco Durán. Aproximación normativa de protección de datos y los derechos de los menores de edad en Colombia Revista Linhas. Florianópolis, v. 17, n. 33, p. 158175, jan./abr. 2016. 


\section{Normative approach for data protection and rights of under-18s in Colombia}

\begin{abstract}
This article aims to problematize some challenges that contemporary society, especially arranged in Colombia, around the preservation of the rights of minors have access to and use of the different devices and technological resources that make part of today's digital world. This article builds on some references submitted by authors who have been addressing the theme here proposed, plus some examples of existing regulatory framework in Colombia, as resources allow, not only to lay the foundations of this argumentative essay but also the horizon proactive in it: the need for progress in developing a more coordinated and aimed at the prevention of the violation of rights, through ignorance or other reasons, are violated in the minors currently regulatory framework in Colombia, as well as the need for greater integration and promotion of a scenario of co-responsibility of all involved in the process of formation of underage social partners, both inside and outside the formal education context, for ensuring a social framework to accompany efficiently and ensure the rights of the population taken as the center of our debate.
\end{abstract}

Keywords: Protection of information; Social networks; Cyberbulling; Minors; Colombia.

\section{Abordagem normativa de proteção de dados e os direitos dos menores de idade na Colômbia}

\begin{abstract}
Resumo
O presente artigo tem como objetivo problematizar alguns desafios que a sociedade contemporânea tem, em especial a colombiana, no que diz respeito à preservação dos direitos que os menores de idade têm em face do acesso e do uso dos diferentes dispositivos e recursos tecnológicos presentes no mundo digital atual. Para tanto, este artigo toma como base algumas referências apresentadas por autores que têm abordado o tema aqui proposto, além de alguns exemplos do quadro normativo vigente na Colômbia, como recursos que permitirão não somente lançar as bases argumentativas deste ensaio, mas também indicar o horizonte propositivo explícito nele, a saber: a necessidade de avançar no desenvolvimento de um quadro normativo mais articulado e orientado à prevenção da violação dos direitos que, por desconhecimento ou outros motivos, se infringem nos menores de idade na atualidade na Colômbia, assim como a necessidade de uma maior integração e promoção de um cenário de corresponsabilidade de todos os agentes sociais vinculados ao processo de formação dos menores de idade, tanto fora como dentro do contexto educativo formal, a fim de garantir um quadro social que acompanhe e vele eficientemente pelos direitos da população, tomada como centro do nosso debate.
\end{abstract}

Palavras-chave: Proteção de dados; Redes sociais; Cyberbullying; Menores de idade; Colômbia. 
La aparición y auge del uso de las redes sociales, pone a disposición de los usuarios de internet y los escenarios digitales actuales una gran diversidad de servicios y/o oportunidades (como por ejemplo: compartir experiencias mediante fotografías, vídeos, páginas web, textos, música o noticias, enviar mensajes privados a otros usuarios, participar en juegos sociales, y comentar el contenido compartido por otros usuarios, entre otros). Sin embargo, autores como Gandasegui (2011), dan cuenta de los diversos riesgos que pueden traer consigo al conjunto de la sociedad contemporánea, en especial en padres y niños(as), quienes deben saber afrontar fenómenos tipificados, a nivel presencial en dichos contextos digitales. Tal es el caso del ciberbulling', la extorsión, la revelación de secretos, las amenazas, el acoso sexual, entre otros delitos y riesgos que adquieren rasgos dinámicos en nuestras sociedades contemporáneas, ante aumento de la complejidad de los potenciales conflictos generados a través de internet y los escenarios digitales actuales.

Según Gandasegui (2011) las TIC permiten un sinnúmero de maneras de injerencia en la intimidad de las personas, razón por la cual la protección de datos personales es hoy día una necesidad manifiesta, no solo, por el surgimiento de nuevos derechos (derecho al honor, la intimidad y la propia imagen, entre otros), sino en el auge de nuevas formas de vulnerar la esfera privada de las personas, ya sea a través de la recogida, el almacenamiento y el control de los datos personales entre otras modalidades.

Bajo el creciente avance de las TIC y las redes sociales, resulta importante contribuir en el debate alrededor del estado y forma de aproximación que debe hacerse alrededor del aseguramiento del derecho a la intimidad y otros derechos que pueden verse en riesgo latentes, ante el surgimiento de los escenarios digitales actuales, en especial, desde la perspectiva de los usuarios de internet, menores de edad.

El uso de Internet, por parte de los menores de edad, trae consigo en la actualidad un serio dilema para la familia, la comunidad y el Estado, en cuanto a la pertinencia de su

\footnotetext{
${ }^{1}$ Autores como Lanzadabal (2011) entienden el cyberbullying como actos de hostigamiento y acoso realizado por compañeros de clases, bajo la mediación de los equipos y recursos tecnológicos en la actualidad.
} 
uso y el carácter prevalente de los riesgos sobre el conjunto de oportunidades que los recursos y equipos TIC pueden traer consigo.

Este trabajo busca reflexionar sobre los riesgos latentes de los entornos virtuales para los menores de edad; haciendo énfasis en la vulneración al derecho a la intimidad, el "grooming”, "bullyng” y “ciberbullyng”. Situaciones que sin duda representan dificultades a la familia, las administraciones públicas y los órganos encargados en la lucha contra este tipo de delitos. Esto, en vista de la presencia de leyes generales y de la necesidad de una nueva generación de normativas ajustadas a los retos que traen consigo la constante evolución y penetración de las TIC en las sociedades contemporáneas. Un proceso que exige la necesidad de afrontar el papel que deben de jugar los padres, tutores, escuelas, empresas y resto de actores de la sociedad civil, para combatir este actual fenómeno.

\section{Metodología}

El abordaje del tema planteado en este artículo, parte de un análisis crítico de la situación actual de los menores de edad en el uso de internet. Para ello, se aprovechó el trabajo de revisión documental realizado en el marco del proyecto "La intervención de las administraciones pública y la cooperación público-privada para la protección de datos personales y otros derechos en riesgo de los menores de edad en la Sociedad Digital", financiado por la Junta de Andalucía de España, liderado por la Universidad de Granada con el apoyo del Observatorio de Educación de la Universidad del Norte en Colombia y otras instituciones académicas españolas. Proyecto que contó también con el financiamiento de Colciencias en Colombia, orientado a la vinculación de un joven investigador, cuyo aporte al proyecto sirvió para la revisión documental tomada en consideración para el desarrollo de este trabajo.

La revisión documental tomada como base para la elaboración este artículo, se hizo desde una perspectiva crítico racional, el cual sirvió para la revisión reflexiva de los aportes conceptuales propuestos por investigadores vinculados al tema aquí abordado; así como de la revisión de la normatividad vigente y de aquellos casos y sentencias 
relacionadas con el tema aquí abordado, en los últimos 5 años (entre 2010 y 2014) en Colombia.

La revisión documental, además de contribuir al análisis crítico del tema propuesto, contribuye a establecer las bases de reflexión que ayuden a determinar el conjunto de aspectos conceptuales y normativos que deben ser tenidos en cuenta al momento de pensar las relaciones entre los menores de edad e internet, en nuestras sociedades contemporáneas, en nuestro caso al interior de la sociedad colombiana.

\section{Menores de edad en internet ¿Cómo lo utilizan?}

El progreso tecnológico ha contribuido al surgimiento de una nueva generación de ciudadanos en nuestras sociedades contemporáneas que tienen ante sí un sinnúmero de retos vinculados con las TIC.

Como bien destaca Castro (2013), en los tiempos contemporáneos, dominados por el creciente auge de medios digitales, los jóvenes surgen como un grupo poblacional con altos niveles de apropiación y uso de los diferentes recursos y equipos TIC dispuestos en la actualidad.

El rápido avance de las TIC y la facilidad de acceso a estas, ha traído consigo que un número mayor de menores de edad, cuenten con una mayor diversidad de opciones de ingreso a los diferentes escenarios digitales dispuestos desde internet. Bringué \& Sádaba (2009), han dado muestra del creciente proceso de penetración de la tecnología en el quehacer diario de los ciudadanos, en especial en los menores de edad, quienes se caracterizan por hacer presencia en las redes sociales. A este respecto Garmendia (2012) señala que las sociedades contemporáneas, bajo el aumento de contenidos dispuestos desde los escenarios digitales actuales, ofrece a los menores de edad, una gran cantidad de oportunidades en diversos aspectos (e.g. mayor aprendizaje, mayor capacidad y conocimiento digital); así como también, mayores posibilidades, al momento de llevar a cabo procesos de intercambio de información con otros grupos de interés.

Autores como Islas (2013) y Said (2013) dan cuenta de la importancia del avance de los dispositivos móviles para tener dar cuenta del incremento en la penetración de 
Internet y del potencial uso que hacen los jóvenes para estar en contacto con amigos y padres; estableciendo mecanismos de micro organización de sus actividades personales. Ello, a través del creciente acceso a recursos tecnológicos orientados a asegurar y aumentar la participación de estos en las redes sociales virtuales, bajo la mediación de los diferentes recursos que hoy disponen para el envío y recepción de mensajes en tiempo real o inmediato (e.g. WhatsApp, entre otras). El escenario aquí delineado, no solo, trae consigo oportunidades de contacto con amigos o personas que pueden compartir intereses comunes; sino también aspectos negativos (e.g. acoso, suplantación de las identidades y uso de la información privada sin autorización, entre otros casos).

Según el Ministerio de las TIC de Colombia (2015), 8 de cada 10 colombianos utiliza internet y el 54\% de estos, lo hacen a diario pasando en promedio 2,6 horas navegando. Ello, mientras se observa un descenso en el empleo de los ordenadores tradicionales de escritorio en dicho país, ante dispositivos de carácter móvil (ordenadores portátiles y celulares inteligentes).

Para el caso colombiano, el contraste existente entre el incremento en el uso de las TIC y la ausencia de una política educativa, orientadas a promover un aumento de las capacidades requeridas para el acceso y manejo oportuno de las TIC, trae consigo un escenario donde las dificultades de aprovechamiento de los dispositivos y recursos tecnológicos actuales en los menores de edad y jóvenes, adquieren dimensiones de mayor preocupación. Ello, si tenemos en cuenta el marcado uso, con fines de ocio, entretenimiento y recreación, que hacen los miembros de este colectivo en la actualidad, desperdiciando estos, a favor de una mayor formación personal (Argango, Brigué \& Sádaba, 2010).

García (2012) destaca una serie de datos relacionados con los riesgos que más preocupan a los padres y educadores ante el avance de las TIC y su impacto en los menores de edad y jóvenes en nuestras sociedades contemporáneas:

Un $44,3 \%$ de los menores conoce algún caso de ciberbullying; y un 16,8\% dice contactar con desconocidos de su misma edad, ya sea por redes sociales ( $82,6 \%$ sobre el total), ya sea por mensajería instantánea $(58,4 \%)$. Quizá lo más alarmante sea que un $27,7 \%$ acepta también a desconocidos, 
un $44,6 \%$ ha recibido la petición de algún desconocido, y que un $10,4 \%$ ha sido el protagonista (no la víctima) de ese ruego. De igual modo, no deja de sorprender que un $23,3 \%$ haya tenido encuentros con desconocidos cara a cara. (2012, p. 77)

Lo expuesto por García (2012), da cuenta de los potenciales riesgos que trae consigo las TIC, si estas no vienen articuladas con marcos formativos y normativos que ayuden a dar una base social que contribuya a reducir el uso inadecuado de las TIC, por parte de los menores de edad y los jóvenes. En este sentido, autores como Rebollo \& Saltor (2013) señalan la necesidad de que exista una legislación que unifique pretensiones y métodos, ofreciendo un conjunto de medios que logren garantizar los derechos y libertades fundamentales en el entorno digital.

Los menores de edad colombianos, al igual que otros existentes a nivel mundial, hacen parte de la creciente sociedad digital que ha venido transformando, las últimas décadas, las bases de los paradigmas de nuestro mundo contemporáneo. Por tal motivo, resulta conveniente avanzar en el proceso de reflexión que dote a los ciudadanos en la actualidad de mecanismos legales y procedimentales que garanticen los derechos y las responsabilidades que tienen estos en los escenarios digitales actuales. Sin embargo la condición de los menores de edad, hace que los ciudadanos en formación posean rasgos especiales en la normativa, lo cual hace que al resto de miembros de nuestras sociedades en actores vigilantes en hacer valer sus derechos y libertades en los diferentes escenarios digitales que hoy irrumpen ante nosotros.

\section{El amparo legal de los menores de edad en internet en Colombia}

El Estado colombiano no ha sido ajeno al desarrollo de las TIC y de las implicaciones que ellas traen consigo en el desarrollo personal y colectivo de los miembros de su sociedad. Por ende, ha venido desarrollando en los últimos años un marco jurídico bastante extenso en lo concerniente al uso de las TIC. Ello con el fin de asegurar la protección de sus ciudadanos, en especial, de los menores de edad. 
Partiendo de la Ley 1581 del $2012^{2}$, la cual se encuentra erigida sobre los artículos 15 y 20 de la Constitución colombiana, se establecen un conjunto de disposiciones orientadas a la protección de los datos personales; garantizándole a los ciudadanos el derecho a conocer, actualizar, rectificar y suprimir las informaciones que se hayan recogido sobre ellas en las bases de datos o archivos. Estas leyes, establecen y amplían el marco general aplicable a todas las bases de datos de entidades públicas y privadas que almacenen y utilicen datos personales, las cuales, previa a esta ley, se encontraban establecidos estos derechos de manera exclusiva a los datos de carácter financiero y comercial.

La normativa vinculada con la Ley 1581 del 2012, a través de su artículo séptimo define a los menores de edad en una categoría especial, prohibiendo el tratamiento de datos personales de los menores de edad salvo aquellos datos públicos. Sin embargo, a través de la sentencia T-260/12, en el que un padre creó que una cuenta de Facebook a nombre de su hija de 4 años de edad sin que ella fuese consciente de ello, le ordenaron a éste cancelarla, ya que la Corte Constitucional colombiana precisó que los tratamientos de información de los menores debían ser hechos para fines que tuviesen en cuenta los interés del menor y cuando estos no constituyesen un riesgo de afectación de sus derechos fundamentales de estos; marcando un importante precedente jurídico alrededor de la adecuada utilización de los datos personales de este protegido conjunto.

En la misma línea, el decreto 1377 del $2013^{3}$ el cual reglamenta la ley 1581 del 2012 establece la corresponsabilidad general de la familia, el Estado y la sociedad en el tratamiento de los datos personales de los menores buscando que se asegure el respeto de los derechos fundamentales de los mismos y para sus efectos los representantes legales de los menores de edad otorgarán la autorización velando por el uso adecuado de los mismos. Decreto que marca una visión desde el Estado donde se reafirma el escenario de co-responsabilidad que tienen todos los actores sociales, incluyendo los padres y

\footnotetext{
${ }^{2}$ COLOMBIA. Ley 1581 del 2012, de 11 de noviembre del 2012, "Por la cual se dictan las disposiciones generales para la protección de datos personales”, Gaceta 078/2011.

${ }^{3}$ COLOMBIA. Decreto 1377 del 2013, de 27 de junio del 2013, "Por medio del cual se reglamenta parcialmente la ley 1581 del 2012” Presidencia de la Republica, Ministerio de comercio, industria y turismo.
} 
madres de los menores de edad al momento de asegurar los derechos de estos en los escenarios digitales actuales.

\section{Los riesgos de los menores de edad en los escenarios digitales actuales}

Según cifras del Departamento Administrativo Nacional de Estadística (DANE), alrededor del $35 \%$ de la población colombiana está conformada por personas menores de 18 años de edad, constituyéndose a su vez como el colectivo que más uso hace de las TIC. Por tanto, resulta preocupante que el $80 \%$ de los contenidos que son consultados en internet correspondan a material pornográfico, material que en muchos casos, involucra a menores de 18 años de edad en un 20\% aproximadamente tomando cifras del ministerio colombiano de las tecnologías de la información y la comunicación (El Espectador, 2014).

De acuerdo con el reporte anual de la Asociación Internacional de Líneas Directas de Internet ${ }^{4}$ (INHOPE, 2014), la cantidad de denuncias recibidas de pornografía infantil ${ }^{5}$ en el mundo han venido incrementándose de manera preocupante, pues en 2010 se habían recibido 24.047 denuncias por pornografía infantil, mientras que en el 2013 aumentaron a 37.404 denuncias. Señalan también en el informe que del total de sitios web con pornografía infantil, el $9 \%$ de las victimas resulta ser menores de 8 años, $76 \%$ preadolescentes ( 9 a 12 años) y 15\% adolescentes (mayores de 13 años); y en 75\% de los casos eran niñas y el $13 \%$ varones $^{6}$.

En Colombia, mediante Ley 765 de $2002^{7}$ se aprobó el protocolo facultativo sobre la venta de niños, la prostitución infantil y la utilización de los niños en la pornografía, el cual resulta ser un instrumento internacional que criminaliza las violaciones de los derechos la infancia y hace particular hincapié en la importancia del fomento de una mayor concientización publica y cooperación internacional en las actividades

\footnotetext{
${ }^{4}$ Organismo internacional que lidera 41 líneas de denuncia de pornografía infantil en 36 países.

${ }^{5}$ Entendiendo esta, como cualquier representación visual, fotos, videos, imágenes de computador creadas, modificadas o montajes realizados en los mismos, de conducta sexual explicita que envuelva a menores.

${ }^{6}$ Para la ley colombiana es considerado menor de edad todo aquel que aún no ha cumplido los dieciocho años.

7 COLOMBIA. Ley 765 de 2002, Diario Oficial No. 44.889, de 5 de agosto de 2002, "Por medio de la cual se aprueba el "Protocolo Facultativo de la Convención sobre los Derechos del Niño relativo a la venta de niños, la prostitución infantil y la utilización de los niños en la pornografía", adoptado en Nueva York, el veinticinco (25) de mayo de dos mil (2000).
} 
encaminadas para combatir estos delitos. A su vez, la Ley 679 de $2001^{8}$ y la Ley 1336 del $2009^{9}$, promulgadas en este país toman medidas de protección contra la explotación, la pornografía, el turismo sexual y demás formas de abuso sexual con menores de edad, estableciendo todo un marco jurídico que incluye prohibiciones, sanciones y hasta cárcel para los proveedores o servidores, administradores y usuarios de redes globales de información, en cuanto a alojar en su propio sitio imágenes, textos, documentos o archivos audiovisuales que impliquen directa o indirectamente actividades sexuales con menores de edad, material pornográfico. Ello, cuando existan indicios de que las personas fotografiadas o filmadas sean menores de edad o se compartan y/o promocionen vínculos o links de sitios web que contengan o distribuyan material pornográfico relativo a menores de edad.

De acuerdo con lo expuesto por Niño \& Nuñez (2009), Colombia está expuesta ante un creciente uso de las TIC con fines pornográficos:

Un dato indicativo de este uso de las TIC es que una búsqueda sencilla en Google sobre "pornografía en Colombia" arroja 458.000 resultados, cuando se ingresa "sexo turismo en Colombia" los resultados ascienden a 1.220.000. (2009, p. 42)

Información que permite evidenciar la gran cantidad de material con contenido pornográfico que está circulando en la red en la cual nuestros menores de edad, no solo pudiesen estar fungiendo como espectadores, pero también como víctimas de estos delitos, los cuales deben ser tenidos en cuenta a nivel social como uno de los argumentos de paso para garantizar los mecanismos que aseguren el acompañamiento idóneo de estos, en el proceso de alfabetización que están llevando a cabo, a partir de la diversidad de dispositivos y recursos TIC a su alcance en su actualidad.

\footnotetext{
${ }^{8}$ COLOMBIA. Ley 679 de 2001, 3 de agosto de 2001, "Por medio de la cual se expide un estatuto para prevenir y contrarrestar la explotación, la pornografía y el turismo sexual con menores, en desarrollo del artículo 44 de la Constitución Política", publicado en diario oficial No. 44509.

${ }^{9}$ COLOMBIA. Ley 1336 de 2009, de 21 de julio de 2009, "Por medio de la cual se adiciona y robustece la Ley 679 de 2001, de lucha contra la explotación, la pornografía y el turismo sexual con niños, niñas y adolescentes", publicado en Diario Oficial No. 47417.
} 
Otro de los riesgos que trae consigo internet y el creciente aprovechamiento de los recursos y dispositivos tecnológicos a favor de diferentes actos de acoso dirigido a los menores de edad. Al respecto, Smith (2006) da cuenta de uno que en los últimos años ha venido adquiriendo relevancia dentro del debate académico y social, a saber: el ciberbullying o ciberacoso, el cual debe ser entendido como todo acto agresivo e intencionado que es llevado a cabo de manera repetitiva y constante a lo largo del tiempo, mediante el uso de formas de contacto electrónicas por parte de un grupo o de un individuo contra una víctima que no puede defenderse fácilmente. En este sentido, el ciberbullying (acoso realizado bajo la medición tecnológica) comparte con el bullying (acosos tradicional) elementos propios de un desequilibrio de poder, frecuencia e intencionalidad, y a veces o en la gran mayoría de los casos resulta ser un comportamiento producto o continuidad del bullyng desarrollado en el ambiente escolar. Unos actores que autores como Collell \& Esqudè (2006) han destacado el gran impacto que trae consigo ambos, en especial el ciberbulling, al permitir el uso internet su uso anónimo, convirtiéndolo en un incognito que trastorna y fragmenta con crueldad la vida personal de sus pares.

El anonimato que permiten los escenarios digitales actuales, dan oportunidades al engaño, la coacción y el acoso a menores de edad, por parte de uno o más adultos, dando lugar a su vez a lo que hoy día se conoce con el nombre de "Grooming" o una forma de acoso, que tiene su inicio en los espacios personales como (blogs, chats, redes sociales, páginas de juegos, entre otros), los cuales son frecuentados en su mayoría por niños y adolescentes. Situación que es aprovechada por adultos (agresores) que buscan establecer una relación y un control emocional sobre el menor (víctima), con fines usualmente orientados a los sexuales (COLLELL \& ESQUDÉ, 2008).

Es el anonimato, por tanto, uno de los principales riesgos que debe asumirse ante el avance de las TIC. Hecho que, si bien no estimamos debe ser causal de argumentos orientados a restringir o sacar máximo provecho de los dispositivos y recursos tecnológicos dispuestos en la actualidad por nuestros menores de edad, si resulta importante tenerlo presente para llevar a cabo acciones avocadas a garantizar el marco de derechos que estos tienen como parte activa de nuestras sociedades, los cuales deben ser asumidos como especiales, en vista del desarrollo humano que poseen. Hecho que 
amerita reiterar de nuevo el importante rol que tienen todos los agentes sociales en favor de garantizar el marco de aplicación de las normas y procedimientos que ayuden a asegurar lo aquí expuesto.

\section{Internet como herramienta educativa}

El papel que juegan las TIC en las sociedades contemporáneas es fundamental, ya que contribuye a la generación de procesos de socialización que inciden en nuestros menores de edad y jóvenes, en general (e.g. estilo de vida, actividad laboral y desarrollo escolar, entre otros aspectos). Sin embargo, el avance de la tecnología, como ha quedado plasmado en el apartado anterior, también ha traído consigo la multiplicación de los escenarios de riesgo en esta población, trayendo consigo situaciones que afectan emocionalmente a estos. (LAPLACETTE, Juan Augusto; BECHER, Carolina; FERNANDEZ, Silvana; GÓMEZ, Lucía Alejandra; LANZILLOTTI, Alejandra; LARA, Lorena Aldana; 2011: p 316-319)

La Ley 1620 del $2013^{10}$ en Colombia o más conocida como la Ley de Convivencia Escolar, tiene por objeto enfrentar las distintas manifestaciones de agresiones y violencia que vulneran los derechos de los estudiantes en escuelas y colegios. Esta normativa, mediante la promoción y el fortalecimiento de la formación ciudadana pretende minimizar los índices de acoso escolar (tradicionales o los ejercidos desde los escenarios digitales actuales), estableciendo un marco normativo que pretende la creación de comités de convivencia que garanticen la protección de los derechos y la vigilancia de las normas establecidas los diferentes manuales de convivencias dispuestos en los escenarios de enseñanza y en la ley colombiana.

Algo que resulta destacable de la Ley 1620 del 2013 es que no deja recaer la responsabilidad únicamente en las entidades educativas, pues le asigna varias responsabilidades a los padres de familia, al ser garantes del derecho que tienen los

\footnotetext{
${ }^{10}$ COLOMBIA. Ley 1620 del 2013, de 15 de marzo de 2013, "Por el cual se crea el sistema nacional de convivencia escolar y formación para los derechos humanos, la educación para la sexualidad y la prevención y mitigación de la violencia escolar", publicado en diario oficial No.087/2012.
} 
menores de edad al momento de vivir en un ambiente de calidez, seguridad y cariño dentro del hogar. Para ello, estos deben acompañar de manera permanente y activa a sus hijos en todo el proceso pedagógico adelantado en la escuela, además de asumir responsabilidades en actividades que propendan al aprovechamiento del tiempo libre de sus hijos para el desarrollo de competencias ciudadanas.

Sin embargo, muy a pesar de que contamos con normatividad suficiente que, de cierta manera, regula gran mayoría de situaciones de riesgo que encuentran los menores de edad en la red, resulta pertinente señalar que estas normas han sido producto de coyunturas que obligaron a legislar en estos aspectos. Por tal motivo, terminan convirtiéndose en legislación dispersa que en ocasiones resulta ser ineficiente en la buscada garantía de los derechos de los menores de edad en internet, de forma articulada.

Hoy en día, estudios como los realizados por Subrahmanyam, Reich, Waecheter \& Espinoza (2008), concluyen que los menores de edad hacen uso de Internet y más concretamente de las herramientas de comunicación, de forma complementaria a la comunicación presencial, coincidiendo mayoritariamente su red de contactos presenciales con la red de contactos en que encuentran en la misma red. Por tanto, como bien es señalado por Díaz \& Pinto (2014):

Uno de los retos para de la sociedad actual está encaminado a utilizar Internet como herramienta educativa, no quedarse con el manejo instrumental de TIC, sino convertir este nuevo escenario en condiciones favorables para diseñar y ejecutar metodologías y prácticas innovadoras. (2014, p. 4)

Es por ello que es muy importante que las administraciones públicas, los directivos docentes, docentes y padres de familia contribuyan a implementar procesos que incentiven las comprensiones que rodean las nuevas formas de aprender, bajo la mediación de las TIC. Ello, a través del diseño de procesos que enriquezca actividades, tareas y experiencias llevados a cabo a través de los diferentes recursos y dispositivos tecnológicos, por parte de los menores de edad, tanto en las escuelas como en el núcleo familiar y en los diferentes escenarios sociales donde llevan a cabo sus diferentes 
actividades diarias (DÍAZ \& PINTO, 2014). A este respecto, Pérez (2013) señala que la sociedad de la información, en general, y las TIC, en particular, incide de manera significativa en todos los niveles del mundo educativo, puesto que:

Las nuevas generaciones van asimilando de manera natural esta nueva cultura que se va conformando y que para nosotros conlleva muchas veces importantes esfuerzos de formación, de adaptación y de "desaprender" muchas cosas que ahora "se hacen de otra forma" o que simplemente ya no sirven (2013, p. 6).

El uso de las TIC en los procesos educativos trae consigo todo un cambio de paradigma que nos invita a aprovechar todo el conjunto de posibilidades proporcionadas por estas herramientas. Sin embargo, como señala Rodríguez (2014) es pertinente una real y total alfabetización digital, no exclusivamente con los estudiantes, sino de todo el conjunto de la sociedad (la familia, el Estado, la comunidad). Ello, con el fin de llevar a cabo procesos que mejoren la productividad y la mejora de los procesos de enseñanza y aprendizaje y con ello la gestión de entornos educativos más integrales. Pero sobre todo, se hace necesario integrar políticas públicas y medidas educativas que aporten a los menores de edad, caracterizados como especial por su no mayoría de edad, de todos los elementos informativos y recursos normativos que velen por estos, de forma preventiva, a través de un contexto marcado por un mayor conocimiento de los potenciales peligros asociados al uso de las tecnologías, animando a los infantes a asumir la responsabilidad de su propia seguridad tanto como sea posible para beneficiarse mejor de estos entornos y saber hacer frente mejor a los riesgos latentes en la Red.

\section{Conclusiones}

Colombia es un país con un complejo entramado normativo con relación al uso de las TIC y la prevención de los posibles riesgos que el uso inadecuado de estás puedan significar para los menores. Sin embargo, es una legislación dispersa y muy amplia, quedándose en ocasiones corta ante situaciones puntuales. Ello sin dejar de lado el 
carácter no preventivo del marco normativo vigente, ya que en la gran mayoría de los casos se aplica cuando el constituye en una clara evidencia de un vacío institucional que contribuye a no dar garantías suficientes sobre los derechos que jurídico que amparan a los menores de edad en la actualidad, ante el avance de las TIC, pues la norma vigente se encuentra latente pero no tiene su desarrollo, aplicabilidad y resultados esperados, precisamente dada la carencia de especialidad y unificación de esta. (LAPLACETTE, Juan Augusto; BECHER, Carolina; FERNANDEZ, Silvana; GÓMEZ, Lucía Alejandra; LANZILLOTTI, Alejandra; LARA, Lorena Aldana; 2011: p. 316-319)

A pesar de todos los riesgos que subsisten en el mundo digital, como por ejemplo los indicados por García (2012), Gandasegui (2011), Smith (2006) o Collell \& Esqudé (2008), entre otros autores tomados como referentes en este artículo, y más aun de los que faltan por ser identificados, teniendo en cuenta que el mundo digital es tan dinámico y los avances en estos escenarios se dan de forma muy rápida y cada vez más compleja, satanizar internet o cohibir de su uso a los menores significa un grave error, ya que las TIC también se presentan como una importante herramienta colaborativa que, no solo, deben ser aprovechada para la optimización de los procesos comunicativos o como medio para desarrollar relaciones interpersonales, sino además debe verse como herramienta útil en los procesos de enseñanza y aprendizaje.

Las TIC y su rápido avance han significado que cada vez sea mayor el número de usuarios que hacen uso de estas herramientas (PÉREZ, 2013). Sin embargo, el uso que le estamos dando a estas herramientas no siempre es el adecuado. Hecho que trae consigo múltiples consecuencias a quienes emplean inadecuadamente los dispositivos y recursos TIC en la actualidad, en especial en el caso de los menores de edad. Por tanto, resulta preocupante la nueva generación de riesgos que deben asumir los usuarios, en especial los que aquí nos centramos en tratar (menores de edad), de los recursos digitales actuales, puesto que pueden convertirse en blancos fáciles de diversas modalidades de ataques y vulneraciones a las cuales se exponen en estos contextos digitales. Ello, como resultado del flujo de información, intercambio y contacto que se generan desde la amplia diversidad de escenarios digitales en la actualidad, cuyos rasgos característicos están en la rapidez, inmediatez y en el potencial uso anónimo de los datos, cuyos rastros dejamos de forma voluntaria o no (en muchos casos), mientras navegamos en internet. 
Muchos usuarios en internet aún no han tomado conciencia de la totalidad de impactos generados que se pueden tener a partir de la difusión de una acción desde los recursos dispuestos en dicho escenario, mucho menos teniendo en cuenta lo que se refiere a la vulneración de los derechos que como ciudadanos tienen. Por tanto resulta pertinente bajo todo este auge tecnológico, contribuir en el debate alrededor del estado y forma de aproximación que debe hacerse alrededor del aseguramiento de los diversos derechos, en especial, desde la perspectiva de los usuarios de internet menores de edad y de los diferentes escenarios digitales que irrumpen en él.

El avance de las TIC hace necesario involucrarse en todo este proceso que implica la construcción de un ambiente más seguro para nuestros menores de edad, con respecto al uso de los diferentes dispositivos y recursos que hacen parte de ello. Sobre todo si tenemos presente los retos expuesto por Díaz \& Pinto (2014). Pero esta labor no solo debe venir del Estado o centros de enseñanza, sino también desde los hogares, los cuales son los espacios iniciales de formación de nuestros menores de edad, para sacar máximo provecho de los escenarios digitales actuales. Por ello, resulta clave educarnos como sociedad para poder educar a los menores de edad, con el fin de brindarles la información necesaria para que entiendan de los riesgos a los que se encuentran expuestos y brindarles las herramientas y el acompañamiento necesario para lograr combatirlos.

A pesar de lo expuesto en el párrafo anterior, las administraciones públicas (Estado) tiene ante sí la tarea de desarrollar todo un marco legal o normativo orientado a la cimentación de políticas públicas dirigidas a fomentar la alfabetización digital, desde la articulación de los diferentes agentes educativos (profesores, familia, entre otros). Ello con el fin de lograr desarrollar estrategias más efectivas que incentiven el uso adecuado de las TIC, en los términos expuestos por Rodríguez (2014). Sobre todo si tenemos en cuenta la transformación en cómo se están comunicando las nuevas generaciones (Subrahmanyam, Reich, Waecheter \& Espinoza, 2008). 


\section{Referencias}

COLLEL, Jordi \& ESCUDÉ, Carme. Ciberbullying. L'assetjament a través de la xarxa. Àmbits de Psicopedagogia, P.20-23. (2008)

COLLEL, Jordi \& ESCUDÉ, Carme. El acoso escolar: un enfoque psicopatológico. Anuario de Psicologia clinica y de la salud. P. 9-14. (2006).

COLOMBIA. Sentencia T-260/12 (Corte constitucional colombiana 29 de 03 de 2012).

COLOMBIA. Ley 1581 del 2012, de 11 de noviembre del 2012, Por la cual se dictan las disposiciones generales para la protección de datos personales, Gaceta 078/2011.

COLOMBIA. Decreto 1377 del 2013, de 27 de junio del 2013, Por medio del cual se reglamenta parcialmente la ley 1581 del 2012. Presidencia de la Republica, Ministerio de comercio, industria y turismo.

COLOMBIA. Ley 765 de 2002, Diario Oficial No. 44.889, de 5 de agosto de 2002, Por medio de la cual se aprueba el "Protocolo Facultativo de la Convención sobre los Derechos del Niño relativo a la venta de niños, la prostitución infantil y la utilización de los niños en la pornografía", adoptado en Nueva York, el veinticinco (25) de mayo de dos mil (2000).

COLOMBIA. Ley 679 de 2001, 3 de agosto de 2001, Por medio de la cual se expide un estatuto para prevenir y contrarrestar la explotación, la pornografía y el turismo sexual con menores, en desarrollo del artículo 44 de la Constitución Política, publicado en diario oficial No. 44509.

COLOMBIA. Ley 1336 de 2009, de 21 de julio de 2009, Por medio de la cual se adiciona y robustece la Ley 679 de 2001, de lucha contra la explotación, la pornografía y el turismo sexual con niños, niñas y adolescentes, publicado en Diario Oficial No. 47417.

COLOMBIA. Ley 1620 del 2013, de 15 de marzo de 2013, Por el cual se crea el sistema nacional de convivencia escolar y formación para los derechos humanos, la educación para la sexualidad y la prevención y mitigación de la violencia escolar, publicado en diario oficial No.087/2012.

DIAZ, J., \& PINTO, A. Reflexiones a partir de las posibilidades y riesgos de las TIC: caso práctico en la experiencia En TIC Confío en La Guajira-Colombia. Congreso Iberoamericano de Ciencia, Tecnología, Innovación y Educación, (P. 1-14). Buenos Aires. (2014).

GANDASEGUI, Vicente. Mitos y realidades de las redes sociales: Información y comunicación en la Sociedad de la Información. PRISMA, 26. (2011)

GARCIA, Antonio. Comunicación, infancia y juventud. En A. Garcia Jimenez, Internet, menores y adolescentes: una aproximación a su realidad (p.75-98). Barcelona: Universitat Oberta de Catalunya. (2012).

INHOPE. (2014). ANNUAL REPORT 2013- 2014. Amsterdan . 
LAPLACETTE, Juan; BECHER, Carolina; FERNANDEZ, Silvana; GÓMEZ, Lucía Alejandra; LANZILLOTTI, Alejandra; LARA, Lorena Aldana. CIBERBULLYING EN LA ADOLESCENCIA: ANÁLISIS DE UN FENÓMENO TAN VIRTUAL COMO REAL. III Congreso Internacional de Investigación y Práctica Profesional en Psicología XVIII Jornadas delnvestigación Séptimo Encuentro de Investigadores en Psicología del Investigación Séptimo Encuentro de Investigadores en Psicología (págs. 316-319). Buenos Aires: UBA. (2011).

MARQUÉS, Pere. Impacto de las Tic en la educación. 3 c TIC cuadernos de desarrollo aplicados a las TIC. (2013).

MINISTERIO DE LAS TIC, de 05 de marzo de 2014. 8 de 10 colombianos están usando Internet. Disponible en: http://www.mintic.gov.co/portal/604/w3-article-1629.html. Accedido el 13 octubre de 2015.

NIÑO, Luis \& NUÑEZ, Lida. Colombia: violencia contra las mujeres y las tecnologías de información y comunicación ¿ Superando el patriarcado? Asociación para el Progreso de las Comunicación. P.1-47. (2009).

REBOLLO, Lucrecio, \& SALTOR, Carlos. El derecho a la protección de datos en España y Argentina : orígenes y regulación vigente. Madrid: Dykinson. (2013).

RODRIGUEZ, Isabel. Riesgos de la comunicación interactiva en adolescentes: propuesta de alfabetización digital. Revista Científica Vozes dos Vales . (2014).

SAID, Elias. Ciudadania Movil. Apropiacion y participacion de jovenes en sectores vulnerables en colombia. Barranquilla: Universidad del Norte. (2013).

SMITH, Peter. Ciberacoso: Naturaleza y extensiòn de un nuevo tipo de acoso dentro y fuera de la escuela. Congreso Educacion Palma de Mallorca. Mallorca. (2006).

SUBRAHMANYAM, Kaveri.; REICH, Stephanie. ;WAECHTER, Natalia., \& ESPINIZA, Guadalupe. Online and offline social networks: Use of social networking sites by emerging adults. Journal of Applied Developmental Psychology, P. 420-433. (2008).

Universidade do Estado de Santa Catarina - UDESC Programa de Pós-Graduação em Educação - PPGE 